\title{
O Setting Terapêutico na realidade do Psicólogo Hospitalar
}

DOI: $10.22289 / 2446-922 X . V 3 N 2 A 5$

\author{
Résia Silva de Morais ${ }^{1}$ \\ Amanda Flávia Gonçalves Lima \\ Daniele Tavares de Oliveira \\ lan Cesar Pereira \\ Jéssica Cristina Tiago da Silva \\ Laiane dos Reis Custódio \\ Leticia Gomes de Azevedo Soares
}

\section{RESUMO}

Este artigo tem como objetivo apresentar uma revisão integrativa de estudos realizados nos anos 2005 a 2016 visando discutir sobre o setting terapêutico do Psicólogo dentro de uma instituição hospitalar A revisão bibliográfica foi realizada a partir de artigos, dissertações e teses disponibilizados nas bases de dados Scielo, Pepsic e BVS, sendo utilizado o descritor "setting terapêutico", em língua portuguesa. Foram selecionados apenas estudos empíricos realizados no território brasileiro. Os resultados foram apresentados a partir das vivencias de profissionais da Psicologia que atuam dentro dos hospitais. Numa perspectiva geral, os estudos revelaram que mesmo diante de algumas dificuldades, o trabalho do Psicólogo é indispensável para auxiliar no cuidado com o paciente, seus familiares e os funcionários que trabalham dentro dos hospitais. Os estudos mostram ainda que o papel do Psicólogo vai além do seu papel como terapeuta, pois ele faz parte de uma equipe multidisciplinar onde todos precisam ajudar uns aos outros para possibilitar melhores resultados aos usuários do serviço. Observou-se ainda que apesar de ser de extrema importância, existem poucos trabalhos discutindo sobre esse tema.

Palavras-chave: Instituição, Psicólogo, Atuação, Hospitais.

\section{ABSTRACT}

This article aims to present a systematic review of studies conducted in the years 2005 to 2016 to discuss the therapeutic setting of the Psychologist within a hospital institution. The bibliographic review was carried out from articles, dissertations and theses available in Scielo databases, DOAJ, Pepsic and VHL, using the term "therapeutic setting" in Portuguese. Only empirical studies carried out in Brazil were selected. The results were presented based on the

Endereço eletrônico de contato: resiamorais@gmail.com

Recebido em 29/09/2017. Aprovado pelo Conselho Editorial e aceito para publicação em 14/11/2017.

Rev. Psicol Saúde e Debate. Dez., 2017:3(2):53-61. 
experiences of Psychology professionals working within the hospitals. In a general perspective, the studies revealed that even in the face of some difficulties, the work of the Psychologist is indispensable to assist in the care of the patient, his relatives and the employees who work inside the hospitals. The studies also show that the Psychologist's role goes beyond his role as a therapist because he is part of a multidisciplinary team where everyone needs to help each other to enable better results for the users of the service. It was also observed that although it is of extreme importance, there are few papers discussing this topic.

Keywords: Institution, Psychologist, Acting, Hospitals.

\section{INTRODUÇÃO}

Houve um tempo que o paciente era visto como um todo, tanto pela Medicina quanto pela Psicologia, misturando-se a natureza, em combinação com as divindades, nessa época não se diferenciavam filosofia, doenças e nem forças da natureza. As práticas utilizadas para solucionar qualquer irregularidade, eram basicamente focadas em rituais e crenças e somente a partir do século XII, após Descartes Newton assumir o analítico como princípio básico da ciência, é que se separou corpo e alma e assim foi possível identificar a existência de duas forças distintas, provenientes do corpo e do psíquico diante da manifestação da doença (Romano,1999).

Apesar da Psicologia ter chegado para ficar no Brasil em 1956, ela só ganhou espaço na rede hospitalar pública após a VIII Conferência Nacional da Saúde (CNS) em 1986, que foi um evento onde definiram as bases do projeto de Reforma Sanitária Brasileira. Até esse momento a Psicologia se restringia a atuar em instituições ambulatoriais e hospitais de saúde mental (Paulin \& Luzio, 2010).

A origem da psicologia clínica e suas representações foi marcada por alguns aspectos ao longo da história, entre eles o próprio termo clínica que significa à beira do leito, evidencia a presença do modelo médico nessa área de atuação do Psicólogo, trazendo como foco de atenção a compreensão e o tratamento da doença, onde buscam o profissional para apresentar a sua queixa e esperam uma solução rápida e eficaz do seu mal psíquico, comparando um sofrimento causado por desordem do psicológico e do simbólico à doenças físicas que são tratadas através de medicamentos como faz o médico. Esse padrão acabou levando Psicólogos a uma forma de atuação limitada a ambiente fechado por muitos anos, uma relação onde era praticada a escuta daqueles que de certa forma são excluídos e incompreendidos (Dutra, 2004).

Com o passar do tempo o modelo clássico de psicologia clínica já não atendia todas as demandas, pois as necessidades mentais se expandiram para o campo da saúde e dos 
hospitais obrigando os profissionais da Psicologia a se movimentarem e buscar meios de atuar nesses lugares.

A Psicologia da Saúde e a Psicologia Hospitalar possuem semelhanças no que tange às formas de atuação prática dos especialistas dessas áreas. Uma tarefa que pode ser desenvolvida nos campos da psicologia clínica, hospitalar e da saúde é que a Psicologia clínica propõe um amplo trabalho de saúde mental nos três campos de atuação, primário, secundário e terciário, a Psicologia da saúde também abrange nesses níveis, mas é aplicada no âmbito sanitário, priorizando as implicações psicológicas, sociais e físicas da saúde. O que difere a Psicologia Hospitalar das outras duas, é o fato dela limitar-se à instituição-hospital e, tendo como consequência, o trabalho de prevenção secundária e terciaria (Castro \& Bornholdt, 2004).

A necessidade da presença de Psicólogos nos hospitais cresce a cada dia, porém a quantidade de profissionais se disponibilizando para esse tipo de função não é muito animador, desde a formação acadêmica, é possível notar que a preferência é a clínica clássica, pois o modelo que as universidades ensinam durante todo o período letivo, está voltado para esse tipo de atuação. Isso seria um dos fatores responsáveis por essa especialização tão importante estar em falta no mercado, outro fator seria a dificuldade que o profissional dessa área enfrenta para conseguir desempenhar o seu papel institucional, pois é muito frequente que mesmo ocupando a vaga de Psicólogo Hospitalar ele se encontre em setores administrativos exercendo papéis que não coincidem com a sua real finalidade (Almeida \& Malagris, 2015).

O papel do Psicólogo hospitalar consiste em dar apoio aos pacientes e aos seus familiares, isso envolve questões emocionais e pessoais, o que acaba exigindo um manejo de forma diferenciada por parte do profissional, pois acaba limitando processos importantes como o sigilo, fazendo com que em muitos casos seja necessário uma intervenção na presença de outras pessoas e episódios como esse gera uma série de angústias tanto no indivíduo que recebe $o$ atendimento quanto naqueles que acabam presenciando a cena, sendo assim, cabe ao profissional a sensibilidade de encontrar um meio que amenize situações como essas (Azevedo, Morais \& Marafon, 2017). Esse contato direto com os usuários do serviço, nem sempre é desempenhado pelo Psicólogo, muitas vezes tem enfermeiros especializados em saúde mental, ou até mesmo a área da psiquiatria entra em ação. A interdisciplinaridade, ocorre quando vários profissionais atuam em prol da melhora do paciente, e é de extrema importância quando executado com eficiência (Carvalho, \& Lustosa, 2008).

O presente estudo tem como objetivo apresentar uma revisão bibliográfica de Rev. Psicol Saúde e Debate. Dez., 2017:3(2):53-61. 
estudos publicados em português, realizados nos últimos 10 anos (2007 a 2017) visando discutir o setting terapêutico do Psicólogo clínico e institucional, levantando questões que implicam a dificuldade enfrentada para adaptação na transição de uma área para a outra.

\section{MÉTODO}

O estudo parte através do método da revisão integrativa da literatura e que tem como finalidade sintetizar resultados obtidos em pesquisa fornecendo informações mais amplas, permitindo assim, um corpo de conhecimento.

O levantamento do material foi empreendido a partir de artigos, disponibilizados entre 2007 e 2017 nas bases de dados Scielo, Pepsic e BVS, sendo utilizado os descritores "Psicologia Hospitalar, Setting Terapêutico e Psicólogo Clínico" em língua portuguesa.

Inicialmente, foram analisados os títulos dos artigos com o intuito de eliminar referências repetidas, em cada busca. Em seguida, os resumos anexados foram selecionados e uma leitura prévia dos mesmos determinou a seleção do material necessário para as revisões. Para a inclusão dos artigos, foram empregados os seguintes critérios: estudos empíricos realizados no território brasileiro e publicados na íntegra que retrataram, as vivências de um Psicólogo no ambiente institucional, como ele foi inserido nesse meio e as dificuldades enfrentadas para a realização dessa mudança.

Os resultados serão apresentados em forma integrativa do conteúdo conforme as análises dos resultados, discussão e conclusão.

\section{RESULTADOS E DISCUSSÃO}

Foram selecionados 13 artigos, dos quais dois artigos foram usados como base de referência sobre a atuação do psicólogo no contexto hospitalar. O primeiro Azevedo \& Crepaldi (2016), narra que o trabalho do psicólogo no ambiente hospitalar pode ser considerado uma ação ou caminho da psicologia da saúde que está concentrada na atenção terciaria de saúde, com a possibilidade de várias práticas de atividades e intervenções que devem ocorrer de acordo com a abordagem teórica utilizada por cada profissional da psicologia. O mesmo autor relata que o trabalho desempenhado visa o acompanhamento dos pacientes em momentos de adoecimento auxiliando-os na aceitação, adaptação e compreensão do momento que está sendo vivenciado. Deve-se considerar a importância da família e toda a equipe profissional que englobam todo o contexto em que o paciente está vivenciando. Todas essas pessoas que estão neste contexto englobam muitas significações.

Rev. Psicol Saúde e Debate. Dez., 2017:3(2):53-61. 
Além disso, relatam que a atuação acontece baseando-se na promoção de saúde e as intervenções podem acontecer dentro das diversas áreas do contexto hospitalar como: medicação, enfermaria, pediatria, unidades e centros de terapias intensivas, blocos cirúrgicos e outros (Azevedo \& Crepaldi, 2016).

Já para Carvalho (2013), há diversos modelos de atendimentos dentro dos hospitais que se diferem em atuação clínica ou integral. A primeira acontece em um local isolado do hospital em que os pacientes podem ser atendidos individualmente. A atenção integral engloba a família e toda a equipe de profissionais da saúde e o trabalho pode acontecer em qualquer local do hospital não possuindo um local fixo de atendimento. Portanto, é de suma importância que estas intervenções sejam realizadas de forma ética baseada no saber técnico.

Foi encontrado um estudo focando as vivencias de médicos e psicólogos hospitalares. No artigo de Romano (1999), comprovam a necessidade de deixar claro que o paciente não pode ser apenas aquele que é objeto do conhecimento científico onde todos enxerguem a doença e não a pessoa que está doente, é necessário que o paciente entenda o motivo que o levou aquela situação e estar ciente do quadro em que se encontra, para que ele se comprometa a participar diretamente do seu processo de cura, pois só assim essa terá a chance de acontecer, nos casos que não tem solução é esse comprometimento quem ajudará no processo de aceitação do paciente.

Romano (1999), relata que é normal existir comparação do papel desempenhado pelo Psicólogo com o do médico. As funções médicas consistem em diagnosticar um quadro orgânico do paciente, estudar a evolução da doença, visando exclusivamente aspectos físicos, para então fazer um plano de tratamento que atuará no combate dos sintomas apresentados. Já o Psicólogo atua tratando o ser como um todo, questões culturais, emocionais, sociais e toda a simbologia que o envolve. Enquanto a medicina trabalha com a lógica física, a psicologia atua na singularidade individual. Ambos têm participação importante no tratamento da mesma doença, porém, um nos aspectos concretos e outro nos aspectos simbólicos/subjetivo.

Além disso, apesar de serem funções distintas, é de suma importância que esse trabalho seja feito em conjunto, por isso foram criadas as equipes multidisciplinares, compostas por toda a equipe que trabalha dentro do hospital, visando melhor atendimento aos usuários do serviço e até mesmo para melhor execução do trabalho dos que ali atuam. Uma equipe multidisciplinar bem organizada, facilita a cansativa jornada de trabalho, além de colaborar para a diminuição dos agravantes do stress das equipes atuantes (Romano, 1999).

Rev. Psicol Saúde e Debate. Dez., 2017:3(2):53-61. 
Sobre o setting, apenas três artigos foram encontrados. O estudo de Fossi, \& Guareschi (2004) foi realizado em seis hospitais gerais, públicos e particulares da cidade de Porto Alegre, através de uma entrevista com seis psicólogas que atuam no campo hospitalar. Baseado nos depoimentos, alguns relatos apresentaram discussões sobre o setting terapêutico e a atuação de profissionais da área.

Após anos atuando em salas fechadas que favoreciam a construção do vínculo entre terapeuta e paciente, o Psicólogo se viu obrigado a expandir as suas ações para o campo hospitalar, onde não é possível atuar com a mesma tranquilidade que nos consultórios convencionais. Foi necessário que se moldassem de acordo com as necessidades da instituição, dos profissionais que ali atuam e dos usuários que precisam do serviço, ou seja um setting que antes era restrito na relação paciente e terapeuta, agora envolve um número indefinido de participantes. O papel do terapeuta não pode ser mudado em relação a escuta e intervenções, mas é preciso desenvolver técnicas todos os dias para conseguir lidar com as adversidades de cada caso apresentado (Fossi \& Guareschi, 2004).

Segundo Moreira, \& Esteves (2012), na clínica tradicional o conceito setting terapêutico se constitui de regras pré-determinadas e combinações especificas de cada dupla. Sendo marcado e definido por um contrato inicial, onde deve conter os papéis de cada um, deixando claro que poderá acontecer alterações de acordo com o caminhar do tratamento. $O$ terapeuta atua como uma espécie de guardião desse setting para que possa auxiliar no processo durante todo o tratamento. O que nos deixa uma brecha para entender que no contexto hospitalar também possa ter a construção desse setting, onde o profissional encontrará meios para que isso se torne possível, sem deixar o encanto da terapia se perder.

Quando se pensa em tratamento psicológico em espaço aberto, a primeira barreira que é citada é a questão da preservação do sigilo do paciente, tarefa difícil, mas também não é impossível. Apesar das limitações que o setting hospitalar envolve, existem formas onde o profissional da Psicologia dá o suporte necessário para o paciente e seus familiares sem precisar expor os assuntos mais delicados, é verdade que não existe receita pronta para fazer isso funcionar perfeitamente, pois vai depender de fatores que devem ser observados de acordo com o ambiente social o qual o hospital pertence. Uma tarefa delicada que exige um feeling especial por parte do profissional que ali está atuando, pois de maneira alguma se deve colocar a dificuldade na frente de um bom serviço.

Simonetti (2004) afirma em seu estudo que no setting hospitalar o terapeuta não atua considerando apenas as doenças psicológicas classicamente conhecidas como "psicossomáticas", e sim em todas as doenças, pois toda doença traz em si aspectos psicológicos, quando não estão diretamente ligados à sua causa estão relacionados com a

Rev. Psicol Saúde e Debate. Dez., 2017:3(2):53-61. 
própria forma do indivíduo lidar com ela e seu significante, ou seja o foco do Psicólogo hospitalar é o aspecto psicológico em torno do adoecimento. Aspectos esses que são encontrados nos doentes, nos familiares deles e no ambiente em que vivem.

Em Ribeiro \& Morais (2017), o aprimoramento das técnicas de enfrentamento da hospitalização envolve uma série de estratégias em que o profissional pode estar fazendo uso para auxiliar nesse processo dentro do contexto hospitalar de forma que seja possível diminuir o sofrimento do paciente, permitindo que ele expresse verbalmente, fazendo com que ele traga para o concreto aquilo o que ele está vivendo emocionalmente naquele momento para que assim ele consiga ter autonomia e domínio sobre a sua situação. Entre essas técnicas envolve o brincar para a criança, que ao ser introduzida com o cuidado na escolha do brinquedo, pode trazer benefícios muito importantes para que o tratamento apresente melhores resultados.

\section{CONSIDERAÇÕES FINAIS}

Portanto o estudo mostra que a psicologia hospitalar vem ganhando seu espaço e mostrando sua importância, para a promoção e prevenção de doenças físicas e metais. Ao identificar e compreender os problemas emocionais dos pacientes o psicólogo juntamente com os outros profissionais da saúde, ajuda o paciente a enfrentar a doença, diminuindo o sofrimento dele e de sua família.

Saúde física e mental anda juntos, um auxiliando o outro, os dois precisam estarem bem para que o paciente fique bem, papel desenvolvido pelo psicólogo no intuito de ajudar o paciente a lidar e entender o significado do que está acontecendo durante o processo de adoecimento e auxiliando a família a compreender todo esse momento.

O papel do psicólogo hospitalar não e só com o paciente, mas é necessário trabalhar as equipes de saúde e os familiares dos pacientes; ambos necessitados de um suporte psicológico. Ao promover uma boa relação entre equipe profissional, família e paciente, o Psicólogo Hospitalar consegue trabalhar e acolher o problema no todo das relações.

Do mesmo modo, é um trabalho que apresenta muitos obstáculos, pois não está ligado a uma clínica fechada, em um consultório, ela está aberta, e precisa ser flexível e continuar preservando o sigilo mesmo em um espaço institucional.

Essa relação delicada entre paciente e terapeuta que por muitos anos foi a porta fechadas, agora deverá ser feita em campo aberto onde não só o paciente precisa ser cuidado, mas todos que participam direta ou indiretamente daquele cenário. Era esperado que 
esse assunto fosse mais discutido através de estudos, porém ao desenvolver o trabalho apresentado foram encontradas diversas referências sobre a atuação da psicologia no contexto hospitalar, mas muito pouco se encontrou sobre o setting terapêutico no contexto hospitalar sendo este de suma relevância na atuação profissional,

Portanto, mesmo com a pouca exposição do tema e enfrentando muitas dificuldades para se adaptar, o setting terapêutico se mostra de maneira versátil dentro da organização hospitalar, sendo de suma importância manter um ambiente saudável para a intervenção, preservando as questões éticas profissionais mesmo em um ambiente hostil. Onde o principal foco precisa ser o usuário do serviço e as pessoas que ali atuam, sem deixar que a doença passe na frente do indivíduo adoecido.

\section{REFERÊNCIAS}

Almeida, R. A. D., \& Malagris, L. E. N. (2015). Psicólogo da Saúde no Hospital Geral: um Estudo sobre a Atividade e a Formação do Psicólogo Hospitalar no Brasil. Psicologia: Ciência e Profissão, 35(3), 754-767.

Azevedo, A. V. D. S., \& Crepaldi, M. A. (2016). A Psicologia no hospital geral: aspectos históricos, conceituais e práticos. Estudos em Psicologia, 33(4), 573-585.

Azevedo, D., Morais, R., \& Marafon, A. (2017). Importância do Psicólogo na intervenção da Psico-Oncologia em mulheres acometidas pelo câncer de mama. Psicologia e Saúde em debate, 2(Supl. 1), 12-15.

Barros de Carvalho, D. (2013). Psicologia da saúde crítica no contexto hospitalar. Psicologia Ciência e Profissão, 33(2).

Carvalho, M. R. D., \& Lustosa, M. A. (2008). Interconsulta psicológica. Revista da $S B P H, 11(1), 31-47$.

Castro, E. K. D., \& Bornholdt, E. (2004). Psicologia da saúde x psicologia hospitalar: definições e possibilidades de inserção profissional. Psicologia: ciência e profissão, 24(3), 48-57.

Dutra, E. (2004). Considerações sobre as significações da psicologia clínica na contemporaneidade. Estudos de Psicologia, 9(2).

Fossi, L. B., \& Guareschi, N. M. D. F. (2004). A psicologia hospitalar e as equipes multidisciplinares. Revista da SBPH, 7(1), 29-43.

Moreira, L. M., \& Esteves, C. S. (2012). Revisitando a teoria do setting terapêutico.

Paulin, T., \& Luzio, C. A. (2010). A Psicologia na Saúde Pública: desafios para a atuação e formação profissional. Revista de Psicologia da UNESP, 8(2). 
Ribeiro, L., \& Morais, R. (2017). A eficácia da TCC para o enfrentamento da hospitalização em crianças com câncer: uma revisão sistemática. Psicologia e saúde em debate, 2(2), 5875.

Romano, B. W. (1999). Princípios Para a Pratica Da Psicologia Clínica. Casa do psicólogo.

Simonetti, A. (2004). Manual de psicologia hospitalar. Casa do Psicólogo.

Rev. Psicol Saúde e Debate. Dez., 2017:3(2):53-61. 